\title{
Miscellanea
}

REFERENCES

Abramowitz, M. \& Stegun, I. A. (Eds.) (1964). Handbook of Mathematical Functions. Washington; D.C. : National Bureau of Standards.

Barton, D. E. \& Dennis, K. E. (1952). The conditions under which Gram-Charlier and Edgeworth curves are positive definite and unimodal. Biometrika 39, 425-7.

[Received September 1971. Revised March 1972]

\section{On a one-sample distribution-free test statistic $V$}

BY H. CARNAL AND H. RIEDWYL

University of Berne, Switzerland

\section{SUMMary}

A table of exact critical values of a one-sample distribution-free test statistic $V$ is presented for selected significance levels and sample sizes $n=3(1) 20$. It is shown that this test is computationally similar.to the well-known Wilcoxon rank sum test statistic.

Some key words: Distribution-free tests; Wilcoxon test; Empirical distribution function.

\section{INTRODUCTION}

Let $X_{1}, \ldots, X_{n}$ be independent random variables having the continuous distribution function $F(x)$ and let $F_{n}(x)=n^{-1}$ \{number of $j$ with $\left.X_{j}<x\right\}$ be the empirical distribution function. Let $x_{i}$ be a real number with $F\left(x_{i}\right)=i / r$. We define

$$
V=\sum_{i=1}^{n-1} n\left\{F_{n}\left(x_{i}\right)-F\left(x_{i}\right)\right\}=\sum_{i=1}^{n-1}\left\{n F_{n}\left(x_{i}\right)-i\right\}
$$

Table 1. Table of critical values $V$ for one-sided and two-sided tests

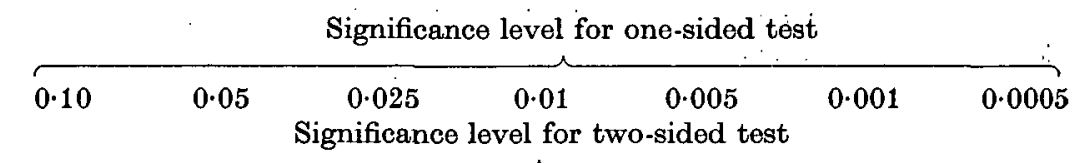

\begin{tabular}{ccccccc}
\hline $0 \cdot 20$ & $0 \cdot 10$ & $0 \cdot 05$ & $0 \cdot 02$ & $0 \cdot 01$ & $0 \cdot 002$ & $0 \cdot 001$ \\
3 & 3 & - & - & - & - & - \\
4 & 5 & 5 & 6 & 6 & - & - \\
5 & 6 & 7 & 8 & 9 & 10 & 10 \\
6 & 8 & 9 & 10 & 11 & 13 & 14 \\
8 & 10 & 11 & 13 & 14 & 16 & 17 \\
9 & 12 & 14 & 16 & 17 & 20 & 21 \\
11 & 14 & 16 & 19 & 21 & 24 & 25 \\
13 & 16 & 19 & 22 & 24 & 28 & 30 \\
15 & 18 & 21 & 25 & 28 & 32 & 34 \\
16 & 21 & 24 & 29 & 31 & 37 & 39 \\
18 & 23 & 27 & 32 & 35 & 42 & 44 \\
20 & 26 & 30 & 36 & 39 & 46 & 49 \\
23 & 29 & 34 & 40 & 44 & 52 & 55 \\
25 & 31 & 37 & 44 & 48 & 57 & 60 \\
27 & 34 & 40 & 48 & 52 & 62 & 66 \\
29 & 37 & 44 & 52 & 57 & 68 & $\mathbf{7 2}$ \\
32 & 40 & 48 & 56 & 62 & 73 & 78 \\
34 & 43 & 51 & 61 & 67 & 79 & 84
\end{tabular}


which is equal to $-n S_{n}^{*}$ proposed by Riedwyl (1967) and give a table of exact critical values of $V$ for significance levels $0.0005,0 \cdot 001,0 \cdot 005,0 \cdot 01,0.025,0.05,0.10$ and sample sizes $3 \leqslant n \leqslant 20$. Since $V\left\{\frac{1}{12} n\left(n^{2}-1\right)\right\}^{-\frac{1}{2}}$ is asymptotically normal with zero mean and variance 1 , one can use normal approximations.

\section{Computation OF THE Distribution OF $V$}

Let $\phi_{g}(x)$ be the indicator function of the set $\left\{x \mid x>X_{j}\right\}$, so that

and let

$$
n F_{n}(x)=\sum_{j=i}^{n} \phi_{j}(x)
$$

$$
V_{j}=\sum_{i=1}^{n-1}\left\{\phi_{j}\left(x_{i}\right)-(i / n)\right\}
$$

so that $V=\sum_{j=1}^{n} V_{j}$. Since $X_{f}$ lies with probability $1 / n$ in each of the intervals $\left(-\infty, x_{1}\right),\left[x_{1}, x_{2}\right), \ldots$, $\left[x_{k-1}, x_{k}\right), \ldots,\left[x_{n-1}, \infty\right), V$, will be, with probability $1 / n$, equal to

$$
\sum_{i=1}^{k-1}\left(-\frac{i}{n}\right)+\sum_{i=k}^{n-1}\left(1-\frac{i}{n}\right)=(n-k)-\frac{1}{2}(n-1) \quad(k=1, \ldots, n) .
$$

Since the $V_{j}$ 's are independent, the distribution of $V$ is the $n$th convolution power of a uniform distribution on the set $\left\{-\frac{1}{2}(n-1),-\frac{1}{2}(n-1)+1, \ldots,-\frac{1}{2}(n-1)+m, \ldots, \frac{1}{2}(n-1)\right\}$.

In Table 1 the exact critical values at $V$ are given for selected significance levels. The characteristic function of $V\left\{\frac{1}{12} n\left(n^{2}-1\right)\right\}^{-\frac{1}{2}}$ is easily computed to be

as $n \rightarrow \infty$.

$$
\begin{aligned}
\phi_{n}(t) & =\left[\frac{\sin \left\{\left(\frac{3 n}{n^{2}-1}\right)^{\frac{1}{2}} t\right\}}{n \sin \left\{\left(\frac{3}{n\left(n^{2}-1\right)}\right)^{\frac{1}{2}} t\right\}}\right]^{n} \\
& =\left\{1-\frac{t^{2}}{2 n}+O\left(n^{-2}\right)\right\}^{n} \\
& \rightarrow e^{-\frac{1}{2} t^{2}}
\end{aligned}
$$

\section{Approximation}

For sample sizes $n>20$, one approximates the distribution of $V$ by the normal distribution. Using a continuity correction, we have

$$
z=\frac{|V|-\frac{1}{2}}{\left\{\frac{1}{12} n\left(n^{2}-1\right)\right\}^{3}} .
$$

Table 2 demonstrates the good agreement already for $n=10$.

Table 2. Exact and normal approximate values of $\operatorname{pr}(V \geqslant k)$ for $n=10$

$\begin{array}{rcc}k & \text { Exact } & \begin{array}{c}\text { Normal } \\ \text { approximation }\end{array} \\ 30 & 0.000324 & 0.000581 \\ 25 & 0.002820 & 0.003495 \\ 20 & 0.015103 & 0.015902 \\ 15 & 0.055552 & 0.055201 \\ 10 & 0.150113 & 0.147799 \\ 5 & 0.312553 & 0.310147 \\ 0 & 0.521623 & 0.521950\end{array}$

\section{Calcutation of $V$}

Let the variables $X_{j}(1 \leqslant j \leqslant n)$ and the quantities $x_{i}(1<i \leqslant n-1)$ of $\S 2$ be ordered according to increasing magnitude and let $\rho_{i}$ be the rank of $x_{i}$ in this ordering. Then we have

$$
V=\sum_{i=1}^{n-1} \rho_{i}-n(n-1) \text {. }
$$


This shows $V$ to be a one-sample analogue of the Wilcoxon (1945) rank sum test statistic, the set of quantiles playing the role of the second sample set.

\title{
5. Example
}

Wetherill (1967, p. 129) tests the one-sided hypothesis that nine observations come from a known normal distribution with mean 40 and standard deviation 1.15. The observations and the underlined quantiles $x_{i}(1 \leqslant i \leqslant n-1)$ with their ranks are, in increasing order,

$$
\begin{aligned}
& \frac{38 \cdot 596}{1}, \frac{39 \cdot 121}{2}, 39 \cdot 4, \frac{39 \cdot 505}{4}, 39 \cdot 6,39 \cdot 8, \frac{39 \cdot 839}{7}, \frac{40 \cdot 161}{8}, 40 \cdot 2, \frac{40 \cdot 495}{10}, \frac{40 \cdot 879}{11}, \\
& 40 \cdot 9, \quad 40 \cdot 9, \quad 41 \cdot 4, \quad \frac{41 \cdot 404}{15}, 41 \cdot 8, \quad 43 \cdot 6 .
\end{aligned}
$$

The $|V|=14$ is just significant at the one-sided significance level of $5 \%$, Table 1 . Without a table we would calculate $z=1.743$ which is also significant compared with the $5 \%$ quantile of the standard cumulative normal distribution. A classical $t=1.91$ on 8 degrees of freedom is significant too $\left(t_{0.95,8}=1.86\right)$.

\section{Discussion}

The test statistic $V$ is an alternative test to competing methods in the one-sample case as the Wilcoxon rank sum test statistic is for the two-sample case. We think that the time used for the calculating will be particularly small.

The authors would like to thank the editor and referee for their helpful suggestions concerning this paper.

\section{REFERENCES}

RrEDWYL, H. (1967). Goodness of fit. J. Am. Statist. Ass. 62, 390-8.

WethermL, G. B. (1967). Elementary Statistical Methods. London: Methuen.

Wricoxon, F. (1945). Individual comparisons by ranking methods. Biometrics 1, 80-3.

[Received January 1971. Revised September 1971]

\section{On the power of Jonckheere's $k$-sample test against ordered alternatives}

\author{
BY ROBERT E. ODEH \\ University of Victoria, British Columbia
}

\section{SUMMARY}

The power of Jonckheere's test for trend is considered for a particular class of nonparametric alternatives. A recursive formula is developed for computing the exact distribution of the test statistic. The mean and variance of the test statistic are derived. An approximation is developed based on the asymptotic distribution of the test statistic. Tables of exact and approximate power are given.

Some key words: Nonparametric test for trend; Jonckheere's test; Power of tests under Lehmann alternatives; Asymptotic distribution of nonparametric tests.

\section{INTRODUCTION}

The test procedure discussed here was proposed by Terpstra (1952) and independently by Jonckheere (1954), but is known as Jonckheere's test in the literature.

Assume that we are given random samples of size $n$ from each of $k$ populations. Denote by $X_{i j}$ the $j$ th observation from the $i$ th population $(i=1, \ldots, k ; j=1, \ldots, n)$. Denote by $F_{i}(\cdot)$ the continuous cumulative distribution function of $X_{i j}$. We wish to test the null hypothesis

$$
H: F_{1}(u)=F_{2}(u)=\ldots=F_{k}(u) \quad(\text { all } u),
$$

